Article

\title{
E-Polytopes in Picard Groups of Smooth Rational Surfaces
}

\author{
Jae-Hyouk Lee ${ }^{1, *}$ and YongJoo Shin ${ }^{2}$ \\ 1 Department of Mathematics, Ewha Womans University, Seoul 03760, Korea \\ 2 Shanghai Center for Mathematical Sciences, Fudan University, Shanghai 103077, China; \\ shinyongjoo@fudan.edu.cn \\ * Correspondence: jaehyouk1@ewha.ac.kr; Tel.: +82-2-3277-3346
}

Academic Editor: Brigitte Servatius

Received: 30 September 2015; Accepted: 13 April 2016; Published: 20 April 2016

\begin{abstract}
In this article, we introduce special divisors (root, line, ruling, exceptional system and rational quartic) in smooth rational surfaces and study their correspondences to subpolytopes in Gosset polytopes $k_{21}$. We also show that the sets of rulings and exceptional systems correspond equivariantly to the vertices of $2_{k 1}$ and $1_{k 2}$ via E-type Weyl action.
\end{abstract}

Keywords: del Pezzo surface; Hirzebruch surface; Gosset polytope; E-polytope

MSC Classifications: 14J26; 14E05

\section{Introduction}

Rational surfaces are complex surfaces birational to $\mathbb{P}^{2}$. According to the classification of surfaces [1], the minimal surfaces of smooth rational surfaces are either a projective plane $\mathbb{P}^{2}$ or a Hirzebruch surface F. In particular, the typical examples of del Pezzo surfaces, which are smooth surfaces $S_{r}$ with the ample anticanonical divisor class $-K_{S_{r}}$, are obtained by blowing up $r(<9)$ points of $\mathbb{P}^{2}$ in general position. Del Pezzo surfaces have drawn the attention of mathematicians and physicists because of their geometries and dualities involving mysterious symmetries. For example, the special divisor classes $l$ (called lines) of a del Pezzo surface $S_{r}$ satisfying $l^{2}=l \cdot K_{S_{r}}=-1$ are bijectively related to the vertices of Gosset polytopes $(r-4)_{21}$, which are one type of semiregular polytopes given by the action of the $E_{r}$ symmetry group (called $E$-polytopes). The well-known 27 lines in a cubic surface $S_{6}$ are bijectively related to the vertices of a Gosset $2_{21}$ obtained by an $E_{6}$-action, and it is well known that the configuration of the 27 lines can also be understood via the action of the Weyl group $E_{6}$ [2,3]. Coxeter [4] applied the bijection between the lines of $S_{6}$ and the vertices in $2_{21}$ to study the geometry of $2_{21}$. The complete list [5] of bijections between lines in del Pezzo surfaces $S_{r}$ and vertices in Gosset polytopes $(r-4)_{21}$ is well known, and the bijections play key roles in many different research fields[6-8]. In particular, the classical application appeared in the study by Du Val [9].

The first author constructed Gosset polytopes as convex hulls in the Picard group $\operatorname{Pic}\left(S_{r}\right)$ and extended the bijections between lines and vertices to correspondences between special divisors in the del Pezzo surface $S_{r}$ and subpolytopes in $(r-4)_{21}$ via the Weyl $E_{r}$-action. correspondences were applied to study the geometry of del Pezzo surfaces and the geometry of Gosset polytopes [10-12].

We consider the special divisor classes $D$ of del Pezzo surfaces $S_{r}$ [10-12], which are rational with self intersection $D^{2}=-2,-1,0,1,2$ (called root, line, ruling, exceptional system, quartic rational divisor, respectively). Here, the rulings of del Pezzo surfaces $S_{r}$ are $\mathbb{P}^{1}$-fibrations of $S_{r}$, and the 
exceptional systems produce rational maps from $S_{r}$ to $\mathbb{P}^{2}$. In fact, one can define these special divisor classes in a smooth projective variety $S$. We define the following special divisor (class) in $\operatorname{Pic}(S)$ :

$$
\begin{aligned}
\mathbf{R}(S) & :=\left\{d \in \operatorname{Pic}(S) \mid d^{2}=-2, d \cdot K_{S}=0\right\} \text { (roots) } \\
\mathbf{L}(S) & :=\left\{l \in \operatorname{Pic}(S) \mid l^{2}=l \cdot K_{S}=-1\right\} \text { (lines) } \\
\mathbf{M}(S) & :=\left\{m \in \operatorname{Pic}(S) \mid m^{2}=0, m \cdot K_{S}=-2\right\} \text { (rulings) } \\
\mathcal{E}(S) & :=\left\{e \in \operatorname{Pic}(S) \mid e^{2}=1, e \cdot K_{S}=-3\right\} \text { (exceptional systems) } \\
\mathcal{Q}(S) & :=\left\{q \in \operatorname{Pic}(S) \mid q^{2}=2, q \cdot K_{S}=-4\right\} \text { (quartic rational divisors) }
\end{aligned}
$$

For each root $d$ in $\mathbf{R}(S)$, we consider a reflection $\sigma_{d}$ on $\left(\mathbb{Z} K_{S}\right)^{\perp}$ in $\operatorname{Pic}(S)$ as defined by

$$
\sigma_{d}(D):=D+(D \cdot d) d \text { for } D \in\left(\mathbb{Z} K_{S}\right)^{\perp}
$$

Since each reflection $\sigma_{d}$ preserves the intersection of divisors and fixes the canonical divisor $K_{S}$, the action of the reflection $\sigma_{d}$ can be extended naturally to the whole Picard group $\operatorname{Pic}(S)$. Therefore, the Weyl group $W(S)$ generated by the reflections on $\left(\mathbb{Z} K_{S}\right)^{\perp}$ acts on $\operatorname{Pic}(S)$, and it acts on each set of special divisors. In this article, we consider the surfaces $S$ whose Weyl groups are of $E_{n}$-type whose extended list is given as follows.

\begin{tabular}{ccccccc}
\hline$n$ & $\mathbf{3}$ & $\mathbf{4}$ & $\mathbf{5}$ & $\mathbf{6}$ & $\mathbf{7}$ & $\mathbf{8}$ \\
\hline$E_{n}$ & $A_{1} \times A_{2}$ & $A_{4}$ & $D_{5}$ & $E_{6}$ & $E_{7}$ & $E_{8}$ \\
\hline
\end{tabular}

For rational surfaces $S$, we add a condition $K_{S}^{2}>0$ so that the intersection is negative definite on each affine hypersurface given as

$$
H_{b}(S):=\left\{D \in \operatorname{Pic}(S) \mid-D \cdot K_{S}=b\right\}
$$

In [10], the first author showed that the set of lines $\mathbf{L}\left(S_{r}\right) \subset H_{1}\left(S_{r}\right)$ of del Pezzo surfaces $S_{r}$ turns out to be an orbit of an $E_{r+4}$-action, and its convex hull in $\operatorname{Pic}\left(S_{r}\right) \otimes \mathbb{Q}$ is the Gosset polytope $(r-4)_{21}$. Therefore, he obtained equivariant correspondences between the special divisors and the subpolytopes in Gosset polytopes and studied the geometry of del Pezzo surfaces, such as the configurations of lines [10-12].

One can ask to extend the class of surfaces from del Pezzo surfaces to rational surfaces (with a condition $K^{2}>0$ on the canonical divisor class $K$ ) and the class of $E$-polytopes from Gosset polytopes related to lines to other $E$-polytopes related to the special divisors. In this article, we give a summary of the first author's works [10-12] on the special divisors of del Pezzo surfaces and the subpolytopes in Gosset polytopes via Weyl actions and provide an extension of the studies on del Pezzo surfaces to the rational surfaces given as the blowing up of Hirzebruch surfaces. We also extend the family of $E$-polytopes from Gosset polytopes of lines to other E-polytopes of special divisors. As a matter of fact, the extension of [10-12] to the blowing up of Hirzebruch surfaces is so natural that most results are parallel to [10-12]. However, there are interesting characterizations different from the studies on del Pezzo surfaces appearing along the monoidal transformation of Hirzebruch surfaces. In this article, we announce the parallel results on fundamental issues without details. The studies related to the monoidal transformation of the blowing up of Hirzebruch surfaces will be explained in [13]. For the extension of families of $E$-polytopes, we consider families of polytopes $2_{k 1}$ and $1_{k 2}$ related to rulings and exceptional systems, respectively. Further studies on correspondences between subpolytopes in $2_{k 1}$ and $1_{k 2}$ and divisors in rational surfaces will be described in other articles. 


\section{Preliminary}

\subsection{Smooth Rational Surfaces with $K^{2}>0$}

In this article, we consider smooth rational surfaces $S$ with the condition $K_{S}^{2}>0$. By the classification of surfaces [1], each smooth rational surface has a minimal surface, a projective plane $\mathbb{P}^{2}$, or a Hirzebruch surface $\mathbf{F}$. In this article, we only consider the blow-ups of the projective plane $\mathbb{P}^{2}$ and the Hirzebruch surface $\mathbf{F}$ in general position. In fact, the studies of the blow-ups of the Hirzebruch surface $\mathbf{F}$ in general position have not done much so that there are no typical descriptions of the general positions of points in F. Below, we give a description of the blow-ups of the Hirzebruch surface $\mathbf{F}$ in general position matching our purpose.

First, we consider the smooth rational surface $S$ with $K_{S}^{2}>0$ whose minimal surface is $\mathbb{P}^{2}$. We have a birational morphism $\rho: S \longrightarrow \mathbb{P}^{2}$ decomposed by contractions $\rho_{j}: S_{j} \longrightarrow S_{j-1}$, $j=1,2, \ldots, r$ of $(-1)$-curves $e_{j}^{\prime}$ (i.e., $\rho=\rho_{1} \circ \cdots \circ \rho_{r-1} \circ \rho_{r}$ ), where $S_{r}=S$ and $S_{0}=\mathbb{P}^{2}$. We remark that each $e_{j}^{\prime}, j=1,2, \ldots, r$ can be obtained by a blow-up of a point $p_{j}$ on $S_{j-1}$. The blow-ups at points $p_{j}, j=1,2, \ldots, r$ allow infinitely near ones on $\mathbb{P}^{2}$. When the points $p_{j}, j=1,2 \ldots, r$ on $\mathbb{P}^{2}$ are in general position, we call $S$ a del Pezzo surface with a degree $K_{S}^{2}=9-r>0$ whose anticanonical divisor $-K_{S}$ is ample. We denote by $e_{j}, j=1,2, \ldots, r-1$ the total transforms of $(-1)$-curves $e_{j}^{\prime}$ by $\rho_{j+1} \circ \cdots \circ \rho_{r-1} \circ \rho_{r}$, and $e_{r}:=e_{r}^{\prime}$. Then, the Picard group of $S$ is generated by $h$ and $e_{j}, j=1,2, \ldots, r$ (i.e., $\left.\operatorname{Pic}(S)=\mathbb{Z} h \oplus \mathbb{Z} e_{1} \oplus \cdots \oplus \mathbb{Z} e_{r}\right)$, where $h$ is a pull-back of a line in $\mathbb{P}^{2}$ by $\rho$. Note that the canonical divisor $K_{S} \equiv-3 h+e_{1}+e_{2}+\cdots+e_{r}$, and $r \leq 8$ since $K_{S}^{2}=9-r>0$.

Also, we deal with a smooth rational surface $X$ with $K_{X}^{2}>0$ whose minimal surface is a Hirzebruch surface $\mathbf{F}$. As above we denote a birational morphism $\rho: X \longrightarrow \mathbf{F}$ decomposed by contractions $\rho_{j}: \mathbf{F}_{j} \longrightarrow \mathbf{F}_{j-1}, j=1,2, \ldots, r$ of $(-1)$-curves $e_{j}^{\prime}$ (i.e., $\rho=\rho_{1} \circ \cdots \circ \rho_{r-1} \circ \rho_{r}$ ), where $\mathbf{F}_{r}=X$ and $\mathbf{F}_{0}=\mathbf{F}$; moreover, we have a map $\varphi^{\prime}: \mathbf{F} \longrightarrow \mathbb{P}^{1}$, which gives a fibration $\varphi:=\varphi^{\prime} \circ \rho: X \longrightarrow \mathbb{P}^{1}$. We denote by $f$ and $s$ a general fibre of $\varphi$ and the special section of $\varphi$ whose self-intersection number is a nonpositive integer $-p$, respectively. We use $\mathbf{F}_{p, r}$ instead of $\mathbf{F}_{r}$ unless there is no confusion. When we consider the contraction $\rho_{j}$ of $e_{j}^{\prime}$ as a (possibly infinitely near) blow-up of a point $p_{j}$ on $\mathbf{F}_{j-1}$, the point $p_{j}$ is located in the special section $s_{j-1}$ on $\mathbf{F}_{j-1}$ or not. If $p_{j}$ is not a point in $s_{j-1}$ then $s_{j}^{2}=s_{j-1}^{2}$. On the other hand, if $p_{j}$ is a point in $s_{j-1}$ then $s_{j}^{2}=s_{j-1}^{2}-1$. Thus, we define that distinct points $p_{j+1}, p_{j+2}, \ldots, p_{r}$ are located on $\mathbf{F}_{-s_{j}^{2}, j}:=\mathbf{F}_{j}$ in general position if each point $p_{k}, k=j+1, \ldots, r$ is not in a special section $s_{j}\left(s_{j}^{2} \neq 0\right)$ and there are no two points $p_{k_{1}}, p_{k_{2}}$ for $k_{1} \neq k_{2} \in\{j+1, \ldots, r\}$ which are in a common general fibre of $\varphi^{\prime} \circ \rho_{1} \circ \rho_{2} \circ \cdots \circ \rho_{j}$. As above, we denote by $e_{j}, j=1,2, \ldots, r-1$ the total transforms of $(-1)$-curves $e_{j}^{\prime}$ by $\rho_{j+1} \circ \cdots \circ \rho_{r-1} \circ \rho_{r}$, and $e_{r}:=e_{r}^{\prime}$. Then the Picard group of $X$ is generated by $f, s$ and $e_{j}, j=1,2, \ldots, r$ (i.e. $\operatorname{Pic}(X)=\mathbb{Z} f \oplus \mathbb{Z} s \oplus \mathbb{Z} e_{1} \oplus \cdots \oplus \mathbb{Z} e_{r}$ ). The canonical divisor $K_{X}$ is written as $K_{X} \equiv(-2-p) f-2 s+e_{1}+e_{2}+\cdots+e_{r}$, and we have $r \leq 7$ since $K_{X}^{2}=8-r>0$.

Remark 1. We can consider an isometric isomorphism $\psi: \operatorname{Pic}\left(\mathbf{F}_{p, r-1}\right) \longrightarrow \operatorname{Pid}\left(S_{r}\right)$ for $r=3,4, \ldots, 8$ defined by

$$
\begin{aligned}
& \left\{\begin{array}{l}
\psi\left(e_{i}\right)=e_{i+1}, 2 \leq i \leq r, \\
\psi\left(e_{1}\right)=h-e_{1}-e_{2}, \\
\psi(f)=h-e_{1}, \\
\psi(s)=\frac{2-p}{2} h+\frac{p}{2} e_{1}-e_{2}
\end{array}\right. \\
& \left\{\begin{array}{l}
\psi\left(e_{i}\right)=e_{i+1}, 1 \leq i \leq r, \\
\psi(f)=h-e_{1}, \\
\psi(s)=\frac{1-p}{2} h+\frac{1+p}{2} e_{1}
\end{array}\right.
\end{aligned}
$$

Then $\psi$ preserves intersection pairings, root systems and the canonical divisor classes between $\operatorname{Pid}\left(\mathbf{F}_{p, r-1}\right)$ and $\operatorname{Pic}\left(S_{r}\right)$. Note the isometric isomorphism in the proof of Lemma 3.2 in [14]. Thus, we can naturally extend the correspondence between the special divisors of a del Pezzo surface $S_{r}$ and the subpolytopes of a Gosset polytope 
to one for $\mathbf{F}_{p, r-1}$. Because the correspondence was established for a del Pezzo surface in [10,12] at first, we use the argument of [10,12] in Sections 3 and 4 to present the original idea, although we have the isometric isomorphism $\psi$.

\subsection{ADE-Polytopes}

The polytopes under consideration in this article are characterized by the highly nontrivial symmetries given by Coxeter groups. Moreover, the polytopes are determined by the corresponding Coxeter-Dynkin diagrams. In this subsection, we introduce the general theory of regular and semiregular polytopes according to their symmetry groups and the corresponding Coxeter-Dynkin diagrams. In particular, we consider a family of semiregular polytopes known as the Gosset polytopes, ( $k_{21}$ according to Coxeter), $2_{k 1}$ and $1_{k 2}$. Here, we only present a brief introduction; for further detail, refer to $[10,15]$.

\subsubsection{Regular and Semiregular Polytopes}

Let $P_{n}$ be a convex $n$-polytope in an $n$-dimensional Euclidean space. For each vertex $O$ of $P_{n}$, when the set of midpoints of all the edges emanating from the vertex $O$ in $P_{n}$ is contained in an affine hyperplane, the set consists of the vertices of an $(n-1)$-polytope. This $(n-1)$-polytope is called the vertex figure of $P_{n}$ at $O$. A regular polytope $P_{n}(n \geq 2)$ is a polytope whose facets and the vertex figure at each vertex are regular. Thus, the facets of a regular $P_{n}$ are all congruent, and the vertex figures are the same. We also call a polytope $P_{n}$ a semiregular one if its facets are regular and the symmetry group of $P_{n}$ acts transitively on the vertices of $P_{n}$. Here we consider two kinds of regular polytopes, a regular simplex and a crosspolytope. A regular simplex $\alpha_{n}$ is an $n$-dimensional simplex with equilateral edges, and a crosspolytope $\beta_{n}$ is an $n$-dimensional polytope whose $2 n$-vertices are the intersections between an $n$-dimensional Cartesian coordinate frame and a sphere centered at the origin. Below we also consider three kinds of families of semiregular polytopes given by Weyl action of $E_{n}$.

\subsubsection{Coxeter-Dynkin Diagrams}

Reflection groups generated by the reflections with respect to hyperplanes (called mirrors) are called Coxeter groups, and the relationships among generating reflections are presented in the Dynkin diagrams of Coxeter groups.

The Coxeter-Dynkin diagrams of Coxeter groups are graphs with labels where their nodes present indexed mirrors and the labels on edges present the order $n$, which is the dihedral angle $\frac{\pi}{n}$ between two mirrors. If $n=2$, namely, two mirrors are perpendicular, we denote it as no edge joining two nodes presenting the corresponding mirrors. This also implies that the corresponding mirrors commute. For $n=3$, since the dihedral angle $\frac{\pi}{3}$ appears very often, we denote it with an edge between two nodes without labels. We only label the edges when the corresponding order is $n>3$. Each Coxeter-Dynkin diagram contains at least one ringed node which represents an active mirror; i.e., we choose a point off the mirrors corresponding to the ringed nodes, and on the mirrors corresponding to the nodes without rings. The construction of a polytope begins with reflecting the point by the active mirrors.

\subsubsection{ADE-Type Coxeter Groups and Isotropy Groups}

We consider Coxeter groups of $A D E$-type, and we call the polytopes given by Coxeter groups of $A D E$-type $A D E$-polytopes. In fact, the Coxeter-Dynkin diagrams of polytopes in this article have only one ringed node and no labeled edges. For these cases, the following simple procedure using the Coxeter-Dynkin diagrams describes possible subpolytopes and gives the total number of them. Each Coxeter-Dynkin diagram of subpolytopes $\tilde{P}$ is the connected subgraph $\Gamma$ of the diagram containing the ringed node. The subgraph obtained by removing all the nodes joined with the subgraph $\Gamma$ represents the isotropy group $G_{\tilde{P}}$ of $\tilde{P}$. Furthermore, the index between the symmetry group $G$ of the ambient polytope and the isotropy group $G_{\tilde{P}}$ gives the total number of such subpolytopes. For example, 
by removing the ringed node, we obtain the subgraph corresponding to the isotropy group of a vertex where the isotropy group is the symmetry group of the vertex figure.

In fact, the complete list of $A D E$-polytopes consists of various polytopes given by $A D E$ Weyl action. However, we consider only a few of them related to the studies in this article as below.

\subsection{4. $A D E$-Polytopes}

(1) (A-polytope) A regular simplex $\alpha_{n}$ is an $n$-dimensional simplex with equilateral edges. Inductively, $\alpha_{n}$ is constructed as a pyramid based on an $(n-1)$-dimensional simplex $\alpha_{n-1}$. The facets of a regular simplex $\alpha_{n}$ are regular simplexes $\alpha_{n-1}$, and the vertex figure of $\alpha_{n}$ is also $\alpha_{n-1}$. For a regular simplex $\alpha_{n}$, only regular simplexes $\alpha_{k}, 0 \leq k \leq n-1$ appear as subpolytopes.

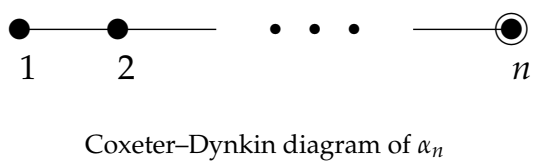

(2) (D-polytope) A crosspolytope $\beta_{n}$ is an $n$-dimensional polytope whose $2 n$-vertices are given as the intersections between an $n$-dimensional Cartesian coordinate frame and a sphere centered at the origin. $\beta_{n}$ is also inductively constructed as a bipyramid based on an (n-1)-dimensional crosspolytope $\beta_{n-1}$, and the $n$-vertices in $\beta_{n}$ form a simplex $\alpha_{n-1}$ if any two vertices are not chosen from the same Cartesian coordinate line. The vertex figure of a crosspolytope $\beta_{n}$ is also a crosspolytope $\beta_{n-1}$, and the facets of $\beta_{n}$ are simplexes $\alpha_{n-1}$. For a crosspolytope $\beta_{n}$, only regular simplexes $\alpha_{k}$, $0 \leq k \leq n-1$ appear as subpolytopes.

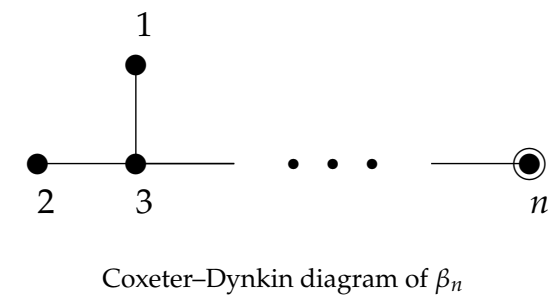

(3) (E-polytope) Gosset polytopes $k_{21}(k=-1,0,1,2,3,4)$ are $(k+4)$-dimensional semiregular polytopes of the Coxeter groups $E_{k+4}$ discovered by Gosset. The vertex figure of $k_{21}$ is $(k-1)_{21}$. For $k \neq-1$ the facets of $k_{21}$-polytopes are the regular simplexes $\alpha_{k+3}$ and the crosspolytopes $\beta_{k+3}$, but all the lower dimensional subpolytopes are regular simplexes. In fact, Coxeter called $4_{21}, 3_{21}$ and $2_{21}$ Gosset polytopes. We extend the list of Gosset polytopes along the extended list of $E_{n}$. Note that a Gosset polytope $(-1)_{21}$, a triangular prism, especially has an isosceles triangle as the vertex figure different from an equilateral triangle.

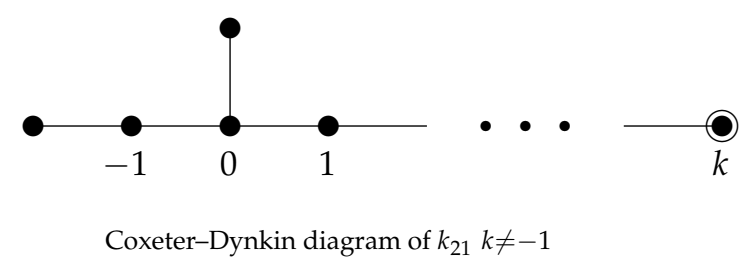

(4) (E-polytope) $2_{k 1}(k=-1,0,1,2,3,4)$ are $(k+4)$-dimensional semiregular polytopes of the Coxeter groups $E_{k+4}$. Here the vertex figure of $2_{k 1}$ is a $(k+3)$-demicube. For $k \neq-1$ the facets of $2_{k 1}$-polytopes are regular simplexes $\alpha_{k+3}$ and semiregular polytopes $2_{(k-1) 1}$. 


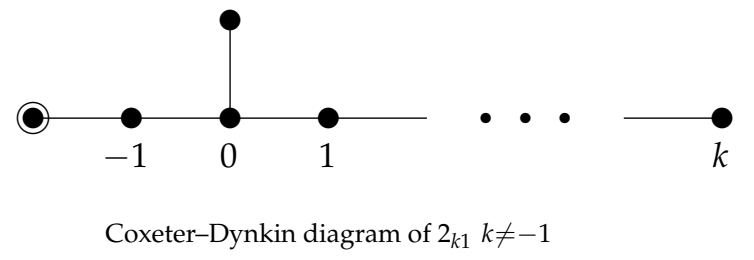

(5) (E-polytope) $1_{k 2}(k=-1,0,1,2,3,4)$ are semiregular polytopes which are $(k+4)$-dimensional polytopes whose symmetry groups are the Coxeter groups $E_{k+4}$. Here the vertex figure of $1_{k 2}$ is a birectified $(k+4)$-simplex. For $k \neq-1$, the facets of $1_{k 2}$-polytopes are the semiregular polytopes $1_{(k-1) 2}$ and $(k+3)$-demicubes, but all the lower dimensional subpolytopes are regular simplexes.

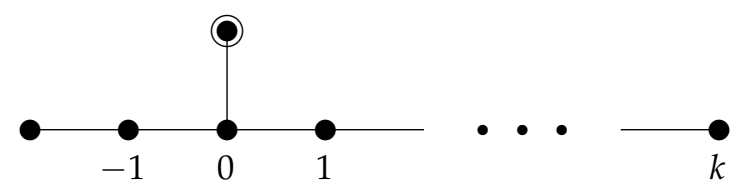

Coxeter-Dynkin diagram of $1_{k 2} k \neq-1$

\subsubsection{Subpolytopes in Gosset Polytopes $k_{21}$}

Below, it is useful to know the total numbers of subpolytopes in $(r-4)_{21}$ for each $r \in\{3,4, \ldots, 8\}$. The numbers of simplexes $\alpha_{i}, i=1,2, \ldots, r-1$ in a Gosset polytope $(r-4)_{21}$ are as follows in Tables 1 and 2.

Table 1. Total numbers of simplexes $\alpha_{i}$ in $(r-4)_{21}$ for $1 \leq \mathrm{i} \leq 7$.

\begin{tabular}{ccccccc}
\hline $\boldsymbol{r}$ & $\mathbf{3}$ & $\mathbf{4}$ & $\mathbf{5}$ & $\mathbf{6}$ & $\mathbf{7}$ & $\mathbf{8}$ \\
\hline $\boldsymbol{\alpha}_{\mathbf{1}}$ & 9 & 30 & 80 & 216 & 756 & 6720 \\
$\boldsymbol{\alpha}_{2}$ & 2 & 30 & 160 & 720 & 4032 & 60,480 \\
$\alpha_{3}$ & 0 & 5 & 120 & 1080 & 10,080 & 241,920 \\
$\boldsymbol{\alpha}_{\mathbf{4}}$ & 0 & 0 & 16 & 648 & 12,096 & 483,840 \\
$\alpha_{5}$ & 0 & 0 & 0 & 72 & 6048 & 483,840 \\
$\alpha_{6}$ & 0 & 0 & 0 & 0 & 576 & 207,360 \\
$\alpha_{7}$ & 0 & 0 & 0 & 0 & 0 & 17,280 \\
\hline
\end{tabular}

The numbers of crosspolytopes $\beta_{r-1}$ in $(r-4)_{21}$ for each $r \in\{3,4, \ldots, 8\}$ are as follows.

Table 2. Total numbers of crosspolytopes $\beta_{r-1}$ in $(r-4)_{21}$.

\begin{tabular}{ccccccc}
\hline $\boldsymbol{r}$ & $\mathbf{3}$ & $\mathbf{4}$ & $\mathbf{5}$ & $\mathbf{6}$ & $\mathbf{7}$ & $\mathbf{8}$ \\
\hline$\beta_{r-1}$ & 3 & 5 & 10 & 27 & 126 & 2160 \\
\hline
\end{tabular}

\subsection{Del Pezzo Surfaces and Gosset Polytopes}

A del Pezzo surface is a smooth rational surface whose anticanonical divisor is ample. All del Pezzo surfaces are $S_{r}$, a blow-up of $\mathbb{P}^{2}$ at $r$ points in general position, for $r=0,1, \ldots, 8$ and $\mathbb{P}^{1} \times \mathbb{P}^{1}$.

To define reflections on $\left(\mathbb{Z} K_{S_{r}}\right)^{\perp}$ in $\operatorname{Pic}\left(S_{r}\right)$, we consider a root system:

$$
\mathbf{R}\left(S_{r}\right)=\left\{d \in \operatorname{Pic}\left(S_{r}\right) \mid d^{2}=-2, d \cdot K_{S_{r}}=0\right\}
$$

With a choice of simple roots

$$
d_{0}=h-e_{1}-e_{2}-e_{3}, d_{i}=e_{i}-e_{i+1}, 1 \leq i \leq r-1
$$


we have a Weyl group $W\left(S_{r}\right)$ of $E_{r}$-type with the Dynkin diagram:

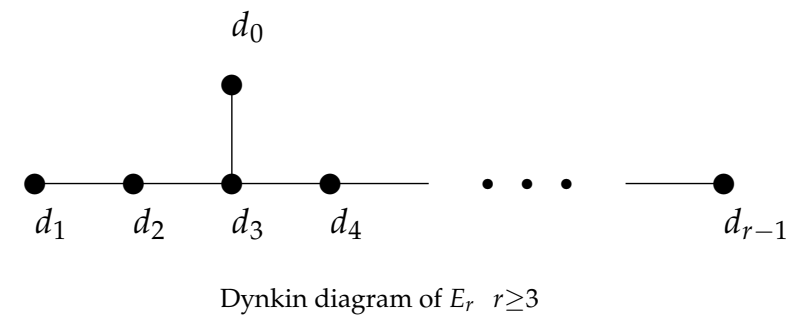

In [10] we obtained correspondences between special divisors on del Pezzo surfaces $S_{r}$ and the faces of Gosset polytopes $(r-4)_{21}$. For this purpose we define affine hyperplane sections in $\operatorname{Pic}\left(S_{r}\right) \otimes \mathbb{Q}$ as

$$
\tilde{H}_{b}\left(S_{r}\right):=\left\{D \in \operatorname{Pic}\left(S_{r}\right) \otimes \mathbb{Q} \mid-D \cdot K_{S_{r}}=b\right\}
$$

where $b$ is a real number.

After fixing a center $\frac{-b}{K_{S_{r}}^{2}} K_{S_{r}}$ in $\tilde{H}_{b}\left(S_{r}\right)$, for each divisor $D \in \tilde{H}_{b}\left(S_{r}\right)$ we obtain

$$
\left(D+\frac{b}{K_{S_{r}}^{2}} K_{S_{r}}\right)^{2}=D^{2}-\frac{b^{2}}{K_{S_{r}}^{2}} \leq 0
$$

and $D \equiv_{\mathbb{Q}} \frac{-b}{K_{S_{r}}^{2}} K_{S_{r}}$ if $\left(D+\frac{b}{K_{S_{r}}^{2}} K_{S_{r}}\right)^{2}=0$ by Hodge index theorem and $K_{S_{r}}^{2}>0$. Therefore, we get a negative definite norm in $\tilde{H}_{b}\left(S_{r}\right)$ induced by the intersection product when we fix a center $\frac{-b}{K_{S_{r}}^{2}} K_{S_{r}}$ in $\tilde{H}_{b}\left(S_{r}\right)$.

Then, by applying reflections defined by the roots for the lines of del Pezzo surfaces $S_{r}$, we obtained the following theorem (in [10]) about the vertices of Gosset polytopes $(r-4)_{21}$ in $\tilde{H}_{1}\left(S_{r}\right) \subset \operatorname{Pic}\left(S_{r}\right) \otimes \mathbb{Q}:$

Theorem 2 (Theorem 4.2 in [10]). For each del Pezzo surface $S_{r}$, the set $\mathbf{L}\left(S_{r}\right)$ of lines on $S_{r}$ is the set of vertices of a Gosset polytope $(r-4)_{21}$ in a hyperplane section $\tilde{H}_{1}\left(S_{r}\right)$.

Remark 3. In fact, the convex hull of the set $\mathbf{L}\left(S_{r}\right)$ of lines in $\tilde{H}_{1}\left(S_{r}\right)$ is the polytope $(r-4)_{21}$.

We consider the set of skew a-lines defined by

$$
\mathbf{L}^{a}\left(S_{r}\right):=\left\{D \in \operatorname{Pic}\left(S_{r}\right) \mid D \equiv l_{1}+l_{2}+\cdots+l_{a} \text { for disjoint lines } l_{i} \text { in } \mathbf{L}\left(S_{r}\right)\right\} .
$$

The following theorem gives a correspondence among the exceptional systems of $S_{r}$, the $(r-1)$-simplexes of $(r-4)_{21}$, and the skew $r$-lines of $S_{r}$.

After we observe the bijections between exceptional systems in del Pezzo surfaces and the top degree simplexes in $(r-4)_{21}$ except for $r=8$, we deduced the following theorem (in [10]).

Theorem 4 (Theorem 5.3 in [10]). When $3 \leq r \leq 8$, each $(r-1)$-simplex in $(r-4)_{21}$ corresponds to an exceptional system in the del Pezzo surfaces $S_{r}$. Moreover, for $3 \leq r \leq 7$, the Weyl group $W\left(S_{r}\right)$ acts transitively on $\mathcal{E}\left(S_{r}\right)$; i.e., the set of exceptional systems in the del Pezzo surface $S_{r}$. Finally $\mathcal{E}\left(S_{r}\right)$ is bijective to $\mathbf{L}^{r}\left(S_{r}\right)$, the set of skew r-lines in $\operatorname{Pic}\left(S_{r}\right)$.

Remark 5. To explain the correspondence, we consider a transformation $\Phi(e)=K_{S_{r}}+3 e$ from $\mathcal{E}\left(S_{r}\right)$ to $\mathbf{L}^{r}\left(S_{r}\right)$ which satisfies

$$
\Phi(e) \cdot K_{S_{r}}=\left(K_{S_{r}}+3 e\right) \cdot K_{S_{r}}=-r, \Phi(e)^{2}=-r
$$

When $r=8$, the set of exceptional systems consists of two orbits. One orbit, with 17280 elements, corresponds to the set of skew 8-lines in $S_{8}$, and the other orbit, with 240 elements, corresponds to the set of E8-roots, because 
each $E_{8}$-root $d$ gives an exceptional system $-3 K_{S_{8}}+2 d$. To distinguish elements of two orbits, we check if $e+3 K_{S_{8}}$ is in $2 \mathrm{Pic}\left(S_{8}\right)$ or not. If so, there is a root satisfying $e+3 K_{S_{8}}=2 d$, which otherwise corresponds to a skew 8-line via $3 e+K_{S_{8}}$.

Also, we had correspondences between rulings of $S_{r}$ and $(r-1)$-crosspolytopes of $(r-4)_{21}$ in [10]:

Theorem 6 (Theorem 5.4 in [10]). For each ruling $f$ in a del Pezzo surface $S_{r}, 3 \leq r \leq 8$, there is a pair of lines $l_{1}$ and $l_{2}$ with $l_{1} \cdot l_{2}=1$ such that $f$ is equivalent to the sum $l_{1}+l_{2}$. Furthermore, the set of rulings in $S_{r}$ is bijective to the set of $(r-1)$-crosspolytopes in $(r-4)_{21}$ and is acted transitively upon by the Weyl group $W\left(S_{r}\right)$.

One can observe that there are two types of $(r-2)$-simplexes in $(r-4)_{21}$. In [12], an $(r-2)$-simplex in $(r-4)_{21}$ is called of A-type if it is contained in an $(r-1)$-simplex in $(r-4)_{21}$, and of $B$-type otherwise. In fact, $(r-2)$-simplexes in $(r-4)_{21}$ form two Weyl orbits according to types and the total numbers of the simplexes of each orbit are as follows in Tables 3 and 4.

Table 3. Total numbers of $(r-2)$-simplexes in $(r-4)_{21}$.

\begin{tabular}{ccccccc}
\hline$(\boldsymbol{r}-\mathbf{4})_{\mathbf{2 1}}$ & $\mathbf{- 1}_{\mathbf{2 1}}$ & $\mathbf{0}_{\mathbf{2 1}}$ & $\mathbf{1}_{\mathbf{2 1}}$ & $\mathbf{2}_{\mathbf{2 1}}$ & $\mathbf{3}_{\mathbf{2 1}}$ & $\mathbf{4}_{\mathbf{2 1}}$ \\
\hline total \# & 9 & 30 & 120 & 648 & 6048 & 207,360 \\
A, B & 3,6 & 10,20 & 40,80 & 216,432 & 2016,4032 & $69,120,138,240$ \\
\hline
\end{tabular}

Table 4. Total numbers of quartic rational divisor classes.

\begin{tabular}{ccccccc}
\hline $\boldsymbol{r}$ & $\mathbf{3}$ & $\mathbf{4}$ & $\mathbf{5}$ & $\mathbf{6}$ & $\mathbf{7}$ & $\mathbf{8}$ \\
\hline total \# & 3 & 10 & 40 & 216 & 2072 & 82,560 \\
I, II & 3,0 & 10,0 & 40,0 & 216,0 & 2016,56 & $69,120,13,440$ \\
\hline
\end{tabular}

For $r=3,4, \ldots, 6$ the set $\mathcal{Q}\left(S_{r}\right)$ of quartic rational divisors consists of one orbit, $W\left(S_{r}\right) \cdot\left(2 h-e_{1}-e_{2}\right)$. However, for $r=7,8$ the set $\mathcal{Q}\left(S_{r}\right)$ consists of two orbits, $W\left(S_{r}\right) \cdot\left(2 h-e_{1}-e_{2}\right) \cup W\left(S_{r}\right) \cdot\left(3 h-\sum_{i=1}^{6} e_{i}+e_{7}\right)$. We say that a quartic rational divisor is of type I if it is in the orbit $W\left(S_{r}\right) \cdot\left(2 h-e_{1}-e_{2}\right)$ and of type II if it is in the orbit $W\left(S_{r}\right) \cdot\left(3 h-\sum_{i=1}^{6} e_{i}+e_{7}\right)$. The total number of such divisor classes in $S_{r}$ is finite and is given in the following table.

Then, we had correspondences between $(r-2)$-simplexes of $A$-type in $(r-4)_{21}$ and quartic rational divisors of type I in $\mathcal{Q}\left(S_{r}\right)$ as follows (in [12]):

Theorem 7 (Theorem 1 in [12]). For disjoint lines $l_{i}, 1 \leq i \leq r-1$ on a del Pezzo surface $S_{r}$, they produce a contraction to $\mathbb{P}^{1} \times \mathbb{P}^{1}$ if there is a quartic rational divisor class $q$ on $S_{r}$ satisfying $2 q+K_{S_{r}} \equiv l_{1}+l_{2}+\cdots+l_{r-1}$. Moreover, the quartic rational divisor classes of type I are bijectively related to $(r-2)$-simplexes of $A$-type in $(r-4)_{21}$.

\section{Special Divisors of Blown-up Hirzebruch Surfaces}

In this section we consider a smooth rational surface $X$ with $K_{X}^{2}>0$ whose minimal surface is a Hirzebruch surface $\mathbf{F}$. For a Hirzebruch surface $\mathbf{F}_{p}$, we allow a blowing up of points in $\mathbf{F}_{p}$ in general position (Note Section 2.1) up to 7 points so that the obtained surface $\mathbf{F}_{p, r}$ satisfies $K_{\mathbf{F}_{p, r}}^{2}>0$. The results in this section for $\mathbf{F}_{p, r}$ are naturally parallel to studies of a del Pezzo surface $S_{r+1}$ (Note Remark 1). The particular and unique characterization on $\mathbf{F}_{p, r}$ comparing to $S_{r+1}$ will be in [13]. 


\subsection{Special Divisors of $\mathbf{F}_{p, r}$}

For $2 \leq r \leq 7$ we consider simple roots of a root system $\mathbf{R}\left(\mathbf{F}_{p, r}\right)$ on $\left(\mathbb{Z} K_{\mathbf{F}_{p, r}}\right)^{\perp}$ in $\operatorname{Pic}\left(\mathbf{F}_{p, r}\right)$ :

$$
\begin{aligned}
& \left\{\begin{array}{l}
d_{0}=e_{1}-e_{2} \\
d_{1}=\frac{p-2}{2} f+s, \\
d_{2}=f-e_{1}-e_{2} \\
d_{i}=e_{i-1}-e_{i}, 3 \leq i \leq r \\
\text { (Note that we have only } \left.d_{0}, d_{1}, d_{2} \text { when } r=2\right)
\end{array}\right. \\
& \left\{\begin{array}{l}
d_{0}=f-e_{1}-e_{2}, \\
d_{1}=\frac{p-1}{2} f+s-e_{1}, \quad \text { if } p \text { is odd } \\
d_{i}=e_{i-1}-e_{i}, 2 \leq i \leq r
\end{array}\right.
\end{aligned}
$$

with a corresponding Coxeter-Dynkin diagram of the type $E_{r+1}$

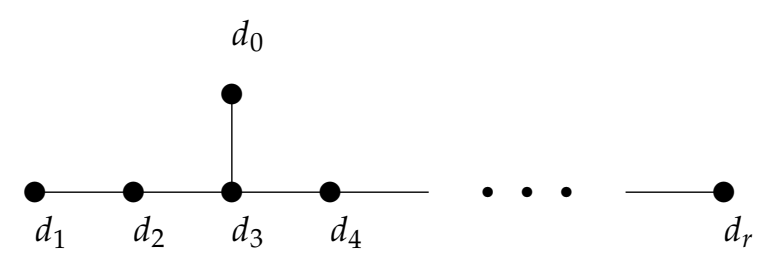

Note that $E_{3}=A_{1} \times A_{2}, E_{4}=A_{4}$ and $E_{5}=D_{5}$. The Weyl group $W\left(\mathbf{F}_{p, r}\right)$ is of the type $E_{r+1}$ for $2 \leq r \leq 7$.

We get a number of special divisors on $\mathbf{F}_{p, r}$ by a simple calculation of the equations of numerical conditions or a coefficient of a theta series of a dual lattice of a root lattice $E_{r+1}$ (see [10]). For $r=2,3, \ldots, 7$, by simple calculation we obtain the total numbers of special divisors in $\mathbf{F}_{p, r}$ which is the same as the one of a del Pezzo surface $S_{r+1}$.

\subsection{Blown-Up Hirzebruch Surfaces and Gosset Polytopes}

We have correspondences between special divisors of a del Pezzo surface $S_{r}$ and faces of a Gosset polytope $(r-4)_{21}$ in Section 2.3. For the special divisors of a blow-up $\mathbf{F}_{p, r}$ of a Hirzebruch surface in general position (Note Section 2.1) and the subpolytopes of a Gosset polytope $(r-3)_{21}$ we can also get parallel correspondences as in the case in Section 2.3 by applying similar proofs in [10]. We define an affine hyperplane $\tilde{H}\left(\mathbf{F}_{p, r}\right)$ and the set $\mathbf{L}^{a}\left(\mathbf{F}_{p, r}\right)$ of skew $a$-lines for $\mathbf{F}_{p, r}$ like the cases of a del Pezzo surface $S_{r}$ (See Section 2.3).

For correspondences between the lines of a blow-up of a Hirzebruch surface and the vertices of a Gosset polytope, we have the following theorem which extends Theorem 2 to Hirzebruch surfaces:

Theorem 8. Let $\mathbf{F}_{r}$ be a blown-up of a Hirzebruch surface in general position for $r \in\{2,3, \ldots, 7\}$. Then the convex hull of the set $\mathbf{L}\left(\mathbf{F}_{r}\right)$ of lines in an affine hyperplane $\tilde{H}_{1}\left(\mathbf{F}_{r}\right)$ is a Gosset polytope $(r-3)_{21}$.

Proof. The lines in $\operatorname{Pic}\left(\mathbf{F}_{r}\right)$ are on the sphere of a radius $-1-\frac{1}{K_{\mathbf{F}_{r}}^{2}}$ with a center $-\frac{1}{K_{\mathbf{F}_{r}}^{2}} K_{\mathbf{F}_{r}}$ in $\tilde{H}_{1}\left(\mathbf{F}_{r}\right)$. Thus a convex hull of $\mathbf{L}\left(\mathbf{F}_{r}\right)$ is a convex polytope in $\tilde{H}_{1}\left(\mathbf{F}_{r}\right)$. We consider the line $e_{r}$ in $\mathbf{L}\left(\mathbf{F}_{r}\right)$. For each simple root $d_{i}, i=0,1, \ldots, r$ in $\mathbf{R}\left(\mathbf{F}_{r}\right)$ explicitly described in the previous subsection, reflections $\sigma_{d_{i}}, i=0, \ldots, r-1$ fix the line $e_{r}$ and the reflection $\sigma_{d_{r}}$ moves the line $e_{r}$ in $\mathbf{L}\left(\mathbf{F}_{r}\right)$. Thus $\sigma_{d_{r}}$ is the only active reflection for the line $e_{r}$. Therefore, the Weyl $E_{r+1}$-orbit of $e_{r}$ in $\operatorname{Pic}\left(\mathbf{F}_{r}\right)$ is the set of the vertices of an $(r-3)_{21}$-polytope obtained by the Coxeter-Dynkin diagram. 


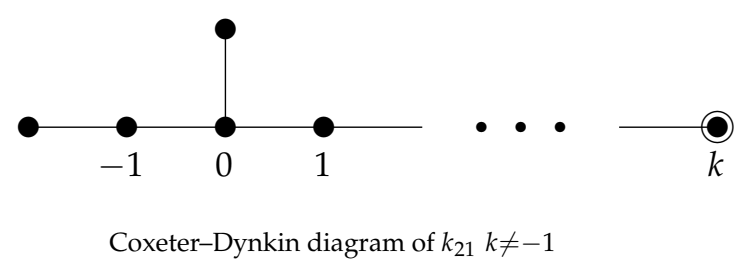

Since $\left|\mathbf{L}\left(\mathbf{F}_{\mathbf{r}}\right)\right|=$ the number of the vertices of an $(r-3)_{21}$-polytope (Table 5), we show that $\mathbf{L}\left(\mathbf{F}_{r}\right)$ is a Weyl $E_{r+1}$-orbit of $e_{r}$ and acted transitively by the Coxeter group of $E_{r+1}$-type. Thus it is the set of the vertices of a $(r-3)_{21}$-polytope. Therefore, we conclude that the convex hull of $\mathbf{L}\left(\mathbf{F}_{r}\right)$ is a Gosset polytope $(r-3)_{21}$ for each $r=2,4, \ldots, 7$.

Table 5. Total numbers of special divisors.

\begin{tabular}{cccccccc}
\hline $\boldsymbol{r}$ & $\mathbf{1}$ & $\mathbf{2}$ & $\mathbf{3}$ & $\mathbf{4}$ & $\mathbf{5}$ & $\mathbf{6}$ & $\mathbf{7}$ \\
\hline $\mathbf{R}\left(\mathbf{F}_{p, r}\right)$ & 2 & 8 & 20 & 40 & 72 & 126 & 240 \\
$\mathbf{L}\left(\mathbf{F}_{p, r}\right)$ & 3 & 6 & 10 & 16 & 27 & 56 & 240 \\
$\mathbf{M}\left(\mathbf{F}_{p, r}\right)$ & 2 & 3 & 5 & 10 & 27 & 126 & 2160 \\
$\mathcal{E}\left(\mathbf{F}_{p, r}\right)$ & 1 & 2 & 5 & 16 & 72 & 576 & 17,520 \\
$\mathcal{Q}\left(\mathbf{F}_{p, r}\right)$ & 1 & 3 & 10 & 40 & 216 & 2072 & 82,560 \\
\hline
\end{tabular}

The following theorem, which extends Theorem 4 to a Hirzebruch surface, gives correspondences among the set $\mathcal{E}\left(\mathbf{F}_{p, r}\right)$ of exceptional systems, the set $\mathbf{L}^{r+1}\left(\mathbf{F}_{p, r}\right)$ of the skew $(r+1)$-lines and the set of the $r$-simplexes $\alpha_{r}$ of a Gosset polytope $(r-3)_{21}$.

Theorem 9. Let $\mathbf{F}_{r}$ be a blow-up of a Hirzebruch surface in general position for $r \in\{2,3, \ldots, 7\}$. Then there is a bijection compatible with the action of Weyl group $W\left(\mathbf{F}_{r}\right)$ between the set $\mathcal{E}\left(\mathbf{F}_{r}\right)$ of exceptional systems in $\operatorname{Pic}\left(\mathbf{F}_{r}\right)$ and the set of the simplexes $\alpha_{r}$ in a Gosset polytope $(r-3)_{21}$. Moreover, for $2 \leq r \leq 6$, the Weyl group $W\left(\mathbf{F}_{r}\right)$ transitively acts on $\mathcal{E}\left(\mathbf{F}_{r}\right)$, and $\mathcal{E}\left(\mathbf{F}_{r}\right)$ is bijective to $\mathbf{L}^{r+1}\left(\mathbf{F}_{r}\right)$, the set of the skew $(r+1)$-lines in $\operatorname{Pic}\left(\mathbf{F}_{r}\right)$.

Remark 10. Similarly to two Weyl orbits in the set $\mathcal{E}\left(S_{8}\right)$ of the del Pezzo surface $S_{8}$, the set $\mathcal{E}\left(\mathbf{F}_{7}\right)$ of exceptional systems of a blown up Hirzebruch surface $\mathbf{F}_{7}$ has two orbits. The set $\mathbf{L}^{8}\left(\mathbf{F}_{7}\right)$ of skew 8-lines in $\mathbf{F}_{7}$ consists of one orbit with 17280 elements. The set of $E_{8}$-roots consists of the other orbit with 240 elements. To distinguish elements of two orbits, we check if $e+3 K_{\mathbf{F}_{7}}$ is in $2 \mathrm{Pic}\left(\mathbf{F}_{7}\right)$ or not. If so, there is a root satisfying $e+3 K_{\mathbf{F}_{7}}=2 d$, which otherwise corresponds to a skew 8-line via $3 e+K_{\mathbf{F}_{7}}$.

We can also obtain correspondences between the set $\mathbf{M}\left(\mathbf{F}_{r}\right)$ of rulings and the set of the crosspolytopes $\beta_{r}$ of a Gosset polytope $(r-3)_{21}$. In the following, we consider correspondences between

$$
\mathbb{L}\left(\mathbf{F}_{r}\right):=\left\{\left(l_{1}, l_{2}\right) \in \mathbf{L}\left(\mathbf{F}_{r}\right) \times \mathbf{L}\left(\mathbf{F}_{r}\right) \mid l_{1} \cdot l_{2}=1\right\} / \sim
$$

and the crosspolytopes of a Gosset polytope $(r-3)_{21}$, where the relation $\left(l_{1}, l_{2}\right) \sim\left(\bar{l}_{1}, \bar{l}_{2}\right)$ is induced by $l_{1}+l_{2} \equiv \bar{l}_{1}+\bar{l}_{2}$ for any $\left(l_{1}, l_{2}\right)$ and $\left(\bar{l}_{1}, \bar{l}_{2}\right)$ in $\mathbf{L}\left(\mathbf{F}_{r}\right) \times \mathbf{L}\left(\mathbf{F}_{r}\right)$. Therefrom we obtain the following theorem which extends Theorem 6 to Hirzebruch surfaces.

Theorem 11. Let $\mathbf{F}_{r}$ be a blow-up of a Hirzebruch surface in general position for $r \in\{2,3, \ldots, 7\}$. Then there is a bijection compatible with the action of Weyl group $W\left(\mathbf{F}_{r}\right)$ between the set $\mathbf{M}\left(\mathbf{F}_{r}\right)$ of rulings in $\operatorname{Pic}\left(\mathbf{F}_{r}\right)$ and the set of the crosspolytopes $\beta_{r}$ in a Gosset polytope $(r-3)_{21}$. Moreover, the Weyl group $W\left(\mathbf{F}_{r}\right)$ transitively acts on $\mathbf{M}\left(\mathbf{F}_{r}\right)$, and each ruling $f$ in $\mathbf{M}\left(\mathbf{F}_{r}\right)$ is linearly equivalent to $l_{1}+l_{2}$ for two lines $l_{1}, l_{2}$ in $\mathbf{L}\left(\mathbf{F}_{r}\right)$ with $l_{1} \cdot l_{2}=1$. 
Proof. Let $\beta_{r}$ be a crosspolytope in a Gosset polytope $(r-3)_{21}$. By Theorem 8 , we have $2 r$ lines in $\operatorname{Pic}\left(\mathbf{F}_{r}\right)$ corresponding to the vertices of $\beta_{r}$. Choose distinct two pairs $\left(l_{1}, l_{2}\right),\left(\bar{l}_{1}, \bar{l}_{2}\right)$ of distinct two lines corresponding two vertices of $\beta_{r}$ which are not in an edge of $\beta_{r}$. For the corresponding center of $\beta_{r}$, we get $l_{1}+l_{2} \equiv \bar{l}_{1}+\bar{l}_{2}$ in $\operatorname{Pic}\left(\mathbf{F}_{r}\right)$. Moreover, we have $l_{1} \cdot \bar{l}_{2}=0$ (resp. $l_{2} \cdot \bar{l}_{2}=0$ ) because vertices corresponding to $l_{1}, \bar{l}_{2}\left(\right.$ resp. $\left.l_{2}, \bar{l}_{2}\right)$ are in an edge of $\beta_{r}$ in $(r-3)_{21}$. Thus we obtain $\bar{l}_{1} \cdot \bar{l}_{2}=1$ and similarly get $l_{1} \cdot l_{2}=1$. We define an injective map as

$$
c r:\left\{\text { crosspolytopes } \beta_{r} \text { in a Gosset polytope }(r-3)_{21}\right\} \longrightarrow \mathbb{L}\left(\mathbf{F}_{r}\right)
$$

by $\operatorname{cr}\left(\beta_{r}\right):=\left(l_{1}, l_{2}\right)$. The Weyl group $W\left(\mathbf{F}_{r}\right)$ is the Coxeter group of the type $E_{r+1}$. By applying Table 5 , we get the theorem.

Note: For the proof of Theorem 11, we apply a fact that for two distinct lines $l_{1}$ and $l_{2}$ in $\mathbf{F}_{r}$, $l_{1} \cdot l_{2}=0$ if and only if the corresponding vertices in $(r-3)_{21}$ are joined by an edge. This fact can be obtained by simple calculations as in the case of del Pezzo surfaces ([10]).

We define a subset $\mathcal{Q}_{I}\left(\mathbf{F}_{p, r}\right)$ of the set $\mathcal{Q}\left(\mathbf{F}_{p, r}\right)$ of quartic rational divisors in $\operatorname{Pic}\left(\mathbf{F}_{p, r}\right)$ as follows:

$$
\mathcal{Q}_{I}\left(\mathbf{F}_{p, r}\right):=\left\{q \in \mathcal{Q}\left(\mathbf{F}_{p, r}\right) \mid 2 q+K_{\mathbf{F}_{p, r}} \equiv l_{1}+\cdots+l_{r} \text { for mutually disjoint lines } l_{i}\right\} .
$$

For $r=2,3, \ldots, 7$ the set $\mathcal{Q}_{I}\left(\mathbf{F}_{p, r}\right)$ consists of one orbit, $W\left(\mathbf{F}_{p, r}\right) \cdot q_{0}$, where $q_{0} \equiv\left(\frac{p+2}{2}\right) f+s$ (resp. $\left(\frac{p+3}{2}\right) f+s-e_{1}$ ) for a nonnegative even integer (resp. a nonnegative odd integer) $p$. Then we obtain correspondence between the set $\mathcal{Q}_{I}\left(\mathbf{F}_{p, r}\right)$ and the subset of simplexes $\alpha_{r-1}$ in a Gosset polytope $(r-3)_{21}$ as the following theorem which extends Theorem 7 for Hirzebruch surfaces:

Theorem 12. Let $\mathbf{F}_{r}$ be a blow-up of a Hirzebruch surface in general position for $r \in\{2,3, \ldots, 7\}$. Then there is a bijection compatible with an action of Weyl group $W\left(\mathbf{F}_{r}\right)$ between the set $\mathcal{Q}_{I}\left(\mathbf{F}_{r}\right)$ and the set of simplexes $\alpha_{r-1}$ of type $A$ which is the set of simplexes $\alpha_{r-1}$ not contained in any simplexes $\alpha_{r}$ in a Gosset polytope $(r-3)_{21}$.

Remark 13. We obtain $\mathcal{Q}\left(\mathbf{F}_{p, r}\right)=\mathcal{Q}_{I}\left(\mathbf{F}_{p, r}\right)$ for $2 \leq r \leq 5$. On the other hand, we have $\left|\mathcal{Q}\left(\mathbf{F}_{p, r}\right)-\mathcal{Q}_{I}\left(\mathbf{F}_{p, r}\right)\right|=56$ for $r=7$, and $\left|\mathcal{Q}\left(\mathbf{F}_{p, r}\right)-\mathcal{Q}_{I}\left(\mathbf{F}_{p, r}\right)\right|=13440$ for $r=8$. Indeed, for each $r \in\{7,8\}$, the set $\mathcal{Q}\left(\mathbf{F}_{p, r}\right)-\mathcal{Q}_{I}\left(\mathbf{F}_{p, r}\right)=W\left(\mathbf{F}_{p, r}\right) \cdot q_{1}$ which consists of one orbit in $\mathcal{Q}\left(\mathbf{F}_{p, r}\right)$ via an action of Weyl group $W\left(\mathbf{F}_{p, r}\right)$, where $q_{1} \equiv(p+2) f+2 s-\sum_{i=1}^{5} e_{i}+e_{6}$. Thus

$$
\mathcal{Q}\left(\mathbf{F}_{p, r}\right)=W\left(\mathbf{F}_{p, r}\right) \cdot q_{0} \dot{\cup} W\left(\mathbf{F}_{p, r}\right) \cdot q_{1} .
$$

\section{E-Polytopes in Picard Groups of Smooth Rational Surfaces}

In the above Theorems 2 and 8, we know that the convex hulls of both $\mathbf{L}\left(S_{r}\right)$, the set of the lines of a del Pezzo surface $S_{r}$, and $\mathbf{L}\left(\mathbf{F}_{r-1}\right)$, the set of the lines of a blow-up Hirzebruch surface $\mathbf{F}_{r-1}$, are Gosset polytopes $(r-4)_{21}$.

Below, we show that the sets of the special divisors are also identified as the vertices of E-polytopes by verifying the corresponding convex hulls are E-polytopes.

\section{1. $2_{k 1}$ and Rulings}

Above, we recall that the set $\mathbf{M}\left(S_{r}\right)$ of rulings in a del Pezzo surface $S_{r}$ and the set of the crosspolytopes in $(r-4)_{21}$ are equivariantly corresponded via $E_{r}$-type Weyl action (Theorem 6), and we state the similar conclusion for the set $\mathbf{M}\left(\mathbf{F}_{r}\right)$ of rulings in blown-up Hirzebruch surfaces (Theorem 11). In the following Table 6, we observe that the bijections between rulings and the crosspolytopes in $(r-4)_{21}$ can be extended to the vertices of $2_{(r-4) 1}$. Moreover, we show that the convex hulls of rulings are E-polytopes $2_{(r-4) 1}$. 
Table 6. Vertices of $2_{(r-4) 1}$, crosspolytopes of $(r-4)_{21}$, and rulings of $S_{r}\left(\mathbf{F}_{r-1}\right)$.

\begin{tabular}{ccccccc}
\hline $\left.\begin{array}{c}\text { Del Pezzo Surface } S_{r} \\
\text { Blown-up } \\
\text { Hirzebruch } \mathbf{F}_{r-1}\end{array}\right)$ & $\begin{array}{c}S_{\mathbf{3}} \\
\left(\mathbf{F}_{\mathbf{2}}\right)\end{array}$ & $\begin{array}{c}\boldsymbol{S}_{\mathbf{4}} \\
\left(\mathbf{F}_{\mathbf{3}}\right)\end{array}$ & $\begin{array}{c}\boldsymbol{S}_{\mathbf{5}} \\
\left(\mathbf{F}_{\mathbf{4}}\right)\end{array}$ & $\begin{array}{c}\boldsymbol{S}_{\mathbf{6}} \\
\left(\mathbf{F}_{\mathbf{5}}\right)\end{array}$ & $\begin{array}{c}S_{\mathbf{7}} \\
\left(\mathbf{F}_{\mathbf{6}}\right)\end{array}$ & $\begin{array}{c}S_{\mathbf{8}} \\
\left(\mathbf{F}_{\mathbf{7}}\right)\end{array}$ \\
\hline rulings & 3 & 5 & 10 & 27 & 126 & 2160 \\
$2_{(r-4) 1}$ & $2_{-11}$ & $2_{01}$ & $2_{11}$ & 221 & 231 & $2_{41}$ \\
vertices of $2_{(r-4) 1}$ & 3 & 5 & 10 & 27 & 126 & 2160 \\
$(r-4)_{21}$ & $-1_{21}$ & $0_{21}$ & $1_{21}$ & 221 & $3_{21}$ & $4_{21}$ \\
midrule $(r-1)$-crosspolytopes & 3 & 5 & 10 & 27 & 126 & 2160 \\
\hline
\end{tabular}

Theorem 14. For $r \in\{3,4,5,6,7,8\}$, the convex hulls of $\mathbf{M}\left(S_{r}\right)$ (resp. $\mathbf{M}\left(\mathbf{F}_{r-1}\right)$ ) in $\tilde{H}_{2}\left(S_{r}\right) \subset$ $\operatorname{Pic}\left(S_{r}\right) \otimes \mathbb{Q}$ (resp. $\left.\tilde{H}_{2}\left(\mathbf{F}_{r-1}\right) \subset \operatorname{Pic}\left(\mathbf{F}_{r-1}\right) \otimes \mathbb{Q}\right)$ are $2_{(r-4) 1}$-polytopes.

Proof. For a del Pezzo surface $S_{r}$, we consider the ruling $h-e_{1} \in \mathbf{M}\left(S_{r}\right) \subset \tilde{H}_{2}\left(S_{r}\right)$ and its Weyl $E_{r}$-orbit. From the Dynkin diagram of $E_{r}$, the reflection $\sigma_{e_{2}-e_{1}}$ given by $d_{1}=e_{2}-e_{1}$ is the only active reflection moving $h-e_{1}$ among the reflections. Thus, the Weyl $E_{r}$-orbit of $h-e_{1}$ in $\operatorname{Pid}\left(S_{r}\right)$ is the set of the vertices of a $2_{(r-4) 1}$-polytope obtained by the following Coxeter-Dynkin diagram.

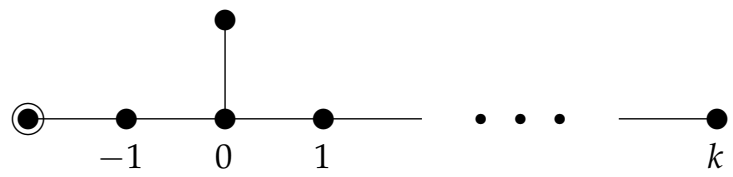

Coxeter-Dynkin diagram of $2_{(r-4) 1} r \neq 3$

Since $\left|\mathbf{M}\left(S_{r}\right)\right|=$ the number of the vertices of a $2_{(r-4) 1}$-polytope (Table 6), we show that $\mathbf{M}\left(S_{r}\right)$ is a Weyl $E_{r}$-orbit of $h-e_{1}$ and transitively acted by the Coxeter group of $E_{r}$-type. Therefore, it is the set of the vertices of a $2_{(r-4) 1}$-polytope. Similarly, for a blown-up of Hirzebruch surface $\mathbf{F}_{r-1}$, we choose the ruling $f$ of $\mathbf{F}_{r-1}$ to get the conclusion. This proves the theorem.

\section{2. $1_{k 2}$ and Exceptional Systems}

As above, we observe that the total numbers of exceptional systems in del Pezzo surfaces $S_{r}$ and the total numbers of the top degree $(r-1)$ simplexes in $(r-4)_{21}$ are the same except for $r=8$. Again in the following Table 7, we observe the bijections between exceptional systems and the $(r-1)$ simplexes in $(r-4)_{21}$ can be extended to the vertices of $1_{(r-4) 2}$. Moreover, we show that the convex hulls of exceptional systems are $1_{(r-4) 2}$. When $r=8$, the set of exceptional systems has two orbits. We show that the convex hull of one of the orbits is $1_{42}$.

Table 7. Vertices of $\left.1_{(} r-4\right) 2,(r-1)$-simplexes in $(r-4)_{21}$ and exceptional systems of $S_{r}\left(\mathbf{F}_{r-1}\right)$.

\begin{tabular}{ccccccc}
\hline $\begin{array}{c}\text { Del } \\
\text { Pezzo Surface } S_{r}\end{array}$ & $S_{\mathbf{3}}$ & $\boldsymbol{S}_{\mathbf{4}}$ & $S_{5}$ & $S_{6}$ & $S_{7}$ & $S_{\mathbf{8}}$ \\
$\left.\begin{array}{c}\text { Blown-up } \\
\text { Hirzebruch } \mathbf{F}_{r-1}\end{array}\right)$ & $\left(\mathbf{F}_{\mathbf{2}}\right)$ & $\left(\mathbf{F}_{\mathbf{3}}\right)$ & $\left(\mathbf{F}_{\mathbf{4}}\right)$ & $\left(\mathbf{F}_{\mathbf{5}}\right)$ & $\left(\mathbf{F}_{\mathbf{6}}\right)$ & $\left(\mathbf{F}_{\mathbf{7}}\right)$ \\
\hline exceptional systems & 2 & 5 & 16 & 72 & 576 & 17,520 \\
$1_{(r-4) 2}$ & $1_{-11}$ & $1_{01}$ & $1_{11}$ & $1_{21}$ & $1_{31}$ & $1_{41}$ \\
vertices of $1_{(r-4) 2}$ & 2 & 5 & 16 & 72 & 576 & 17,280 \\
$(r-4)_{21}$ & $-1_{21}$ & $0_{21}$ & $1_{21}$ & 221 & $3_{21}$ & $4_{21}$ \\
$(r-1)$-simplexes & 2 & 5 & 16 & 72 & 576 & 17,280 \\
\hline
\end{tabular}

Theorem 15. For $r \in\{3,4,5,6,7\}$, the convex hulls of $\mathcal{E}\left(S_{r}\right)$ (resp. $\left.\mathcal{E}\left(\mathbf{F}_{r-1}\right)\right)$ in $\tilde{H}_{3}\left(S_{r}\right) \subset \operatorname{Pic}\left(S_{r}\right) \otimes \mathbb{Q}$ (resp. $\left.\tilde{H}_{3}\left(\mathbf{F}_{r-1}\right) \subset \operatorname{Pic}\left(\mathbf{F}_{r-1}\right) \otimes \mathbb{Q}\right)$ are $1_{(r-4) 2}$-polytopes. 
Proof. For del Pezzo surfaces $S_{r}$, we consider the exceptional system $h \in \mathcal{E}\left(S_{r}\right) \subset \tilde{H}_{3}\left(S_{r}\right)$ and its Weyl $E_{r}$-orbit. From the Dynkin diagram of $E_{r}$, the reflection $\sigma_{h-e_{1}-e_{2}-e_{3}}$ given by $d_{0}=h-e_{1}-e_{2}-e_{3}$ is the only active reflection moving $h$ among the reflections in the Dynkin diagram. Thus the Weyl $E_{r}$-orbit of $h$ in Pic $\left(S_{r}\right)$ is a set of the vertices of an $1_{(r-4) 2}$-polytope obtained by the following Coxeter-Dynkin diagram.

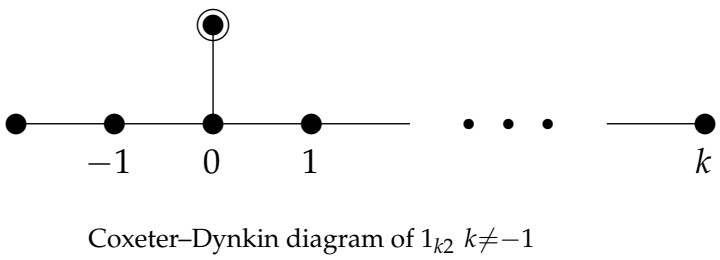

Since $\left|\mathcal{E}\left(S_{r}\right)\right|=$ the number of vertices of an $1_{(r-4) 2}$-polytope, we show that $\mathcal{E}\left(S_{r}\right)$ is the Weyl $E_{r}$-orbit of $h$ and acted transitively by the Coxeter group of $E_{r}$-type. Thus it is the set of the vertices of an $1_{(r-4) 2}$-polytope. Similarly, for a blown-up Hirzebruch surface $\mathbf{F}_{r-1}$, we choose the exceptional system $s+\frac{p+1}{2} f$ for odd $p\left(s+\frac{p+2}{2} f-e_{1}\right.$ for even $\left.p\right)$ of $\mathbf{F}_{r-1}$. Then the only active reflection from the Dynkin diagram is given as $\sigma_{d_{0}}$ where $d_{0}=f-e_{1}-e_{2}$ for odd $p\left(d_{0}=e_{1}-e_{2}\right.$ for even $\left.p\right)$, and we get the conclusion. This proves the theorem.

For $r=8$, there are two orbits of Weyl action in $\mathcal{E}\left(S_{8}\right)$ and the Weyl orbit of $h$ in $\mathcal{E}\left(S_{8}\right)$ is bijectively related to the vertices of $1_{42}$. Also, for $\mathcal{E}\left(\mathbf{F}_{7}\right)$ the Weyl orbit of $s+\frac{p+1}{2} f$ for odd $p\left(s+\frac{p+2}{2} f-e_{1}\right.$ for even $p$ ) is bijectively related to the vertices of $1_{42}$. Therefore, the proof of the above Theorem 15 gives the following Corollary.

Corollary 16. For $r=8$, the convex hull of the Weyl orbit of exceptional system $h$ (resp. $s+\frac{p+1}{2} f$ for odd $p$, $s+\frac{p+2}{2} f-e_{1}$ for even $\left.p\right)$ in $\tilde{H}_{3}\left(S_{8}\right) \subset \operatorname{Pid}\left(S_{8}\right) \otimes \mathbb{Q}\left(\right.$ resp. $\left.\tilde{H}_{3}\left(\mathbf{F}_{7}\right) \subset \operatorname{Pid}\left(\mathbf{F}_{7}\right) \otimes \mathbb{Q}\right)$ is an $E_{8}$-polytope $1_{42}$.

Acknowledgments: The first author was supported by Basic Science Research Program through the National Research Foundation of Korea (NRF) funded by the Ministry of Education, Science and Technology(No.2013R1A1A2012783) and the second author was supported by Shanghai Center for Mathematical Sciences. The authors are grateful to the reviewer for her/his comments, which contributed to improve the quality of the article.

Author Contributions: The article was written by both authors.

Conflicts of Interest: The authors declare no conflict of interest.

\section{References}

1. Beauville, A. Surfaces algébriques complexes; Astérisque, Société Mathématique de France: Paris, France, 1978.

2. Demazure, M. Surfaces de del Pezzo I, II, III, IV, V. In Séminaire sur les Singularités des Surfaces; Springer-Verlag: Berlin, Germany; Heidelberg, Germany; New York, NY, USA, 1980; pp. 21-69.

3. Dolgachev, I.V. Classical Algebraic Geometry. A Modern View; Cambridge University Press: Cambridge, UK, 2012.

4. Coxeter, H.S.M. The polytope 221 , whose twenty-seven vertices correspond to the lines on the general cubic surface. Am. J. Math. 1940, 62, 457-486.

5. Manin, Y. Cubic Forms: Algebra, Geometry, Arithmetic, 2nd ed.; English Translation; North-Holland Mathematical Library 4: Amsterdam, The Netherlands, 1986.

6. Friedman, R.; Morgan, J. Exceptional groups and del Pezzo surfaces. In Symposiumin Honor of C.H. Clemens; Contemp. Math. 312; American Mathematical Society: Providence, RI, USA, 2002; pp. 101-116.

7. Leung, N.C.; Zhang, J.J. Moduli of bundles over rational surfaces and elliptic curves. I. Simply laced cases. J. Lond. Math. Soc. 2009, 80, 750-770.

8. Manivel, L. Configurations of lines and models of Lie algebras. J. Algebra 2006, 304, 457-486.

9. Du Val, P. On the directrices of a set of points in a plane. Proc. Lond. Math. Soc. 1933, 35, $23-74$.

10. Lee, J.H. Gosset Polytopes in Picard Groups of del Pezzo Surfaces. Can. J. Math. 2012, 64, 123-150.

11. Lee, J.H. Configurations of lines in del Pezzo surfaces with Gosset Polytopes. Trans. Am. Math. Soc. 2014, $366,4939-4967$. 
12. Lee, J.H. Contractions of del Pezzo Surfaces to $\mathbb{P}^{2}$ or $\mathbb{P}^{1} \times \mathbb{P}^{1}$. Rocky Mt. J. Math. 2016, in press.

13. Lee, J.H.; Shin, Y. Special Divisor Classes on Blown-up Hirzebruch Surfaces I,II. 2016, preprints.

14. Lahyane, M.; Harbourne, B. Irreducibility of -1-classes on anticanonical rational surfaces and finite generation of the effective monoid. Pac. J. Math. 2005, 218, 101-114.

15. Coxeter, H.S.M. Regular Complex Polytopes, 2nd ed.; Cambridge University Press: Cambridge, UK, 1991.

(C) 2016 by the authors; licensee MDPI, Basel, Switzerland. This article is an open access article distributed under the terms and conditions of the Creative Commons Attribution (CC-BY) license (http://creativecommons.org/licenses/by/4.0/). 